\begin{tabular}{|c|l|}
\hline Title & The metal enrichment of passive gal axies in cosmological simulations of gal axy formation \\
\hline Author(s) & Okamoto, Takashi; Nagashima, Masahiro; Lacey, Cedric G.; Frenk, Carlos S. \\
\hline Citation & $\begin{array}{l}\text { Monthly notices of the royal astronomical society, 464(4), 4866-4874 } \\
\text { https://doi.org/40.1093/mnras/stw2729 }\end{array}$ \\
\hline Issue Date & 2017-02 \\
\hline Doc URL & http://hdl.handle.net/2115/65505 \\
\hline Rights & $\begin{array}{l}\text { This article has been accepted for publication in Monthly notices of the royal astronomical society @ 2016 The Authors } \\
\text { Published by Oxford University Press on behalf of The Royal A stronomical Society. A II rights reserved. }\end{array}$ \\
\hline Type & article \\
\hline File Information & MNRA S464 4866-4874.pdf \\
\hline
\end{tabular}

Instructions for use 


\title{
The metal enrichment of passive galaxies in cosmological simulations of galaxy formation
}

\author{
Takashi Okamoto, ${ }^{1 \star}$ Masahiro Nagashima, ${ }^{2}$ Cedric G. Lacey ${ }^{3}$ and Carlos S. Frenk ${ }^{3}$ \\ ${ }^{1}$ Department of Cosmosciences, Graduates School of Science, Hokkaido University, N10 W8, Kitaku, Sapporo, Hokkaido 060-0810, Japan \\ ${ }^{2}$ Faculty of Education, Bunkyo University, Koshigaya, Saitama 343-8511, Japan \\ ${ }^{3}$ Institute for Computational Cosmology, Department of Physics, Durham University, South Road, Durham DH1 3LE, UK
}

Accepted 2016 October 20. Received 2016 October 20; in original form 2015 October 13

\begin{abstract}
Massive early-type galaxies have higher metallicities and higher ratios of $\alpha$ elements to iron than their less massive counterparts. Reproducing these correlations has long been a problem for hierarchical galaxy formation theory, both in semi-analytic models and cosmological hydrodynamic simulations. We show that a simulation in which gas cooling in massive dark haloes is quenched by radio-mode active galactic nuclei (AGNs) feedback naturally reproduces the observed trend between $\alpha / \mathrm{Fe}$ and the velocity dispersion of galaxies, $\sigma$. The quenching occurs earlier for more massive galaxies. Consequently, these galaxies complete their star formation before $\alpha / \mathrm{Fe}$ is diluted by the contribution from Type Ia supernovae. For galaxies more massive than $\sim 10^{11} \mathrm{M}_{\odot}$, whose $\alpha / \mathrm{Fe}$ correlates positively with stellar mass, we find an inversely correlated mass-metallicity relation. This is a common problem in simulations in which star formation in massive galaxies is quenched either by quasar- or radio-mode AGN feedback. The early suppression of gas cooling in progenitors of massive galaxies prevents them from recapturing enriched gas ejected as winds. Simultaneously reproducing the $[\alpha / \mathrm{Fe}]-\sigma$ relation and the mass-metallicity relation is, thus, difficult in the current framework of galaxy formation.
\end{abstract}

Key words: methods: numerical-galaxies: evolution-galaxies: formation.

\section{INTRODUCTION}

The metal abundance of early-type galaxies provides strong constraints on the theory of their formation. Observational estimates indicate an increase of the overall metal abundance with galaxy luminosity and velocity dispersion (e.g. Faber 1973; Bender, Burstein $\&$ Faber 1993), and also an increase in the average ratio of $\alpha$ elements to iron abundance with luminosity and velocity dispersion (e.g. O'Connell 1976; Worthey, Faber \& Gonzalez 1992; Jørgensen 1999; Thomas et al. 2010; Johansson, Thomas \& Maraston 2012; Conroy, Graves \& van Dokkum 2013).

Theoretical modelling of chemical abundances in elliptical galaxies has been intensively investigated using the so-called monolithic collapse model, in which all of the stars are born in a single burst of short duration, which is usually assumed to be terminated by the ejection of the remaining gas by galactic winds (e.g. Larson 1975; Arimoto \& Yoshii 1987). Studies based on the monolithic collapse model have shown that it is possible to explain the trends of both increasing total metallicity and increasing $\alpha / \mathrm{Fe}$ with galaxy mass (e.g. Matteucci 1994; Thomas, Greggio \& Bender 1999) if the star

^E-mail: okamoto@astro1.sci.hokudai.ac.jp formation time-scale of the initial burst is chosen to have a suitable dependence on galaxy mass.

Models and simulations based on the cold dark matter (CDM) cosmology have naturally reproduced the observed slope of the relation between stellar metallicity and velocity dispersion (or stellar mass) by including strong feedback even in massive galaxies (Kauffmann \& Charlot 1998; Nagashima et al. 2005b; Davé, Oppenheimer \& Finlator 2011; Vogelsberger et al. 2014b). On the other hand, the increase of $\alpha / \mathrm{Fe}$ with velocity dispersion has been a challenge for hierarchical galaxy formation models.

Metal enrichment of elliptical galaxies in the context of hierarchical galaxy formation has been intensively studied by utilizing the so-called semi-analytic models. Thomas (1999) studied the chemical enrichment of elliptical galaxies by considering a closed box model for each galaxy using pre-calculated star formation histories obtained by a semi-analytic model. He claimed that an $\alpha / \mathrm{Fe}$ overabundant population cannot be obtained unless the initial mass function (IMF) is significantly flattened with respect to the Salpeter IMF (Salpeter 1955) during starbursts. Nagashima et al. (2005b) investigated the chemical enrichment of ellipticals by using a semianalytic model in which they solved chemical evolution due to both Type Ia and II supernovae ( $\mathrm{SNe}$ ) self-consistently with galaxy merger trees. Although they reproduced the observed $\alpha$ element 
abundance in ellipticals by assuming a top-heavy IMF in starbursts, their models predict a decreasing $\alpha / \mathrm{Fe}$ ratio with velocity dispersion, $\sigma$.

The role of feedback from active galactic nuclei (AGNs) in chemical enrichment of elliptical galaxies has also been investigated with semi-analytic models. Two distinct modes have been considered for AGN feedback: quasar and radio modes. The former assumes that feedback energy and momentum are proportional to mass accretion rate on to a black hole. Feedback is thus strongest during a rapid growing phase of a black hole (Di Matteo, Springel \& Hernquist 2005). The latter has been introduced to prevent excessive growth of central galaxies in massive haloes from cooling flows. Many semianalytic models assume that the radio mode is triggered by hot gas accretion (e.g. Bower et al. 2006; Croton et al. 2006); this feedback is hence most efficient in massive haloes where the cooling time is longer than the dynamical time. In numerical simulations, these two modes are usually distinguished by the specific accretion rate on to a black hole. When the accretion rate is small, typically less than 1 per cent of the Eddington rate, feedback operates on the radio mode and directly heats the hot halo gas (Sijacki et al. 2007; Okamoto, Nemmen \& Bower 2008). Quasar mode feedback, on the other hand, operates when the specific accretion rate is high and it heats the interstellar medium around the black hole.

De Lucia et al. (2006) demonstrated that, if radio-mode AGN feedback introduced by Croton et al. (2006) is included, massive galaxies have higher metal abundances, older luminosity-weighted ages, and shorter star formation time-scales. De Lucia \& Borgani (2012), however, pointed out that semi-analytic models with radiomode AGN feedback predict decreasing stellar metallicity with increasing stellar mass for massive galaxies, while they can explain old stellar ages of massive galaxies. De Lucia et al. (2006) and De Lucia \& Borgani (2012) appear to reach the opposite conclusions about the effect of AGN feedback on the metallicities in massive galaxies. The more effective feedback in massive galaxies employed by the models in De Lucia \& Borgani (2012) than in De Lucia et al. (2006) presumably causes this discrepancy. Pipino et al. (2009) investigated the $[\alpha / \mathrm{Fe}]-\sigma$ relation in ellipticals by including chemical enrichment both by SNe II and Ia in their semianalytic model. They also considered chemical enrichment by lowand intermediate-mass stars and delay time distribution (DTD) of $\mathrm{SNe}$ Ia. Although they found a better but still marginal agreement with the observed $[\alpha / \mathrm{Fe}]-$ stellar mass relation by taking radio-mode AGN feedback into account, doing this erases a positive correlation between stellar metallicity and velocity dispersion.

Calura \& Menci (2009) implemented a chemical-enrichment model on top of star formation histories obtained from a semianalytic model as done in Thomas (1999). They summed star formation histories over all progenitors of a galaxy in question to compute chemical evolution. They assumed that the IMF becomes top-heavy in systems with star formation rates (SFRs) greater than $100 \mathrm{M}_{\odot} \mathrm{yr}^{-1}$. This assumption, together with quasar-mode AGN feedback and interaction-triggered starbursts, yields a better agreement with the $[\alpha / \mathrm{Fe}]-\sigma$ and mass-metallicity relations than the case where the Salpeter IMF is assumed for all star formation activities.

Calura \& Menci (2011) employed the same model as Calura \& Menci (2009), but computed chemical evolution using individual star formation histories of all progenitors of a galaxy considered. They claimed that inclusion of quasar-mode AGN feedback which quenches late star formation in large haloes and starbursts triggered by galaxy harassment, which boosts star formation activity at high redshift, is crucial and the variable IMF used in Calura \& Menci
(2009) is not necessary to explain the observed $[\alpha / \mathrm{Fe}]-\sigma$ relation, although they did not show the mass-metallicity relation predicted by their model.

Arrigoni et al. (2010) also investigated the $[\alpha / \mathrm{Fe}]-$ mass and mass-metallicity relations by using a semi-analytic model which includes both quasar- and radio-mode AGN feedback and a detailed chemical-evolution model in which they relax the instantaneous recycling approximation. They claimed that a combination of a shallower slope for the IMF and a low SN Ia rate (i.e. a low binary fraction) is required to obtain a good agreement with observations. Yates et al. (2013) implemented three different SN Ia DTDs in their semi-analytic model. They found that a combination of a power-law DTD and metal-rich winds, which drive light $\alpha$ elements directly out of galaxies, gives the best agreement with a wide range of observational data including the $[\alpha / \mathrm{Fe}]-$ mass and mass-metallicity relations. On the other hand, Gargiulo et al. (2015) employed a variable IMF whose slope becomes shallower (more top-heavy) for a higher SFR of a galaxy. They tuned their model to reproduce the observed $[\alpha / \mathrm{Fe}]$-galaxy mass relation, and they claimed that the SFR-dependent IMF is key to reproducing the $[\alpha / \mathrm{Fe}]-$ galaxy mass relation and that a short star formation time-scale due to radio-mode AGN feedback alone cannot explain the slope of the relation.

The numerous semi-analytic studies have shown that it is difficult to simultaneously reproduce the observed $[\alpha / \mathrm{Fe}]-\sigma$ and massmetallicity relations. Different authors propose different solutions, most of which involve some form of AGN feedback, variable IMFs, or a combination of these two.

This problem has not been extensively studied by using cosmological hydrodynamic simulations, which, in principle, have advantages in dealing with chemical enrichment over semi-analytic models, that is (i) chemical enrichment is solved locally in hydrodynamic simulations rather than galaxy wide and (ii) the instantaneous recycling approximation is easily relaxed by using star particles, each of which represents a simple stellar population (SSP), and by solving timed release of mass, metals, and energy from each star particle (e.g. Okamoto et al. 2005).

Some cosmological hydrodynamic simulations also found a decreasing $\alpha /$ Fe ratio with velocity dispersion (Romeo Velonà et al. 2013; Taylor \& Kobayashi 2015). Taylor \& Kobayashi (2015) further showed that quasar-mode AGN feedback can increase $\alpha / \mathrm{Fe}$ in massive galaxies without changing the IMF. Segers et al. (2016b) also showed that $[\alpha / \mathrm{Fe}]$ increases with stellar mass for massive galaxies whose stellar masses are more massive than $10^{10.5} \mathrm{M}_{\odot}$ when AGN feedback is included, while it is roughly constant without AGN feedback.

Recent advances in computer simulations have enabled us to form reasonably realistic galaxy populations in a representative cosmological volume (e.g. Okamoto, Shimizu \& Yoshida 2014; Vogelsberger et al. 2014b; Schaye et al. 2015). In this paper, we investigate the chemical abundances of passive galaxies formed in cosmological hydrodynamic simulations with the physics described by Okamoto et al. (2014, hereafter OSY14), which reproduces key observed properties of galaxy populations, such as the galaxy stellar mass function, SFRs, and gas phase metallicities of star-forming galaxies, for $z \leq 4$. We discuss the role of each physical process in determining the chemical properties of the passive galaxies and reveal successes and limitations of our galaxy formation model.

This paper is organized as follows. In Section 2, we briefly describe our simulations. We present our results in Section 3. The results are summarized and discussed in Section 4. 


\section{SIMULATIONS AND SAMPLE OF GALAXIES}

We study the galaxies formed in cosmological hydrodynamic simulations in the $\Lambda$-dominated CDM $(\Lambda \mathrm{CDM})$ cosmology with the following parameters (Planck Collaboration XVI 2014): $\Omega_{0}=0.318$, $\Omega_{\Lambda}=0.682, \Omega_{\mathrm{b}}=0.049, \sigma_{8}=0.835, n_{\mathrm{s}}=0.962$, and dimensionless Hubble parameter, $h=0.67$. We use a cosmological periodic box of side length $80 \mathrm{~h}^{-1} \mathrm{Mpc}$ for the reference simulation. The simulation code is based on an early version of TREE-PM smoothed particle hydrodynamics (SPH) code GADGET-3. We employ $512^{3}$ dark matter particles and the same number of SPH particles. Each gas particle initially has a mass of $m_{\mathrm{SPH}}^{\text {orig }}=5.2 \times 10^{7} h^{-1} \mathrm{M}_{\odot}$. The gravitational softening length is set to $2.1 h^{-1} \mathrm{kpc}$ in physical units both for dark matter and gas particles after $z=3$.

The baryonic processes and the numerical resolution are exactly the same as in OSY14. We briefly describe the implementation of the feedback and chemical-enrichment processes. Star formation takes place in the dense cold gas, and the star formation time-scale is assumed to be proportional to the local dynamical time as

$\dot{\rho}_{*}=c_{*} \frac{\rho_{\mathrm{gas}}}{t_{\mathrm{dyn}}}$,

where $c_{*}$ and $t_{\mathrm{dyn}}$ are, respectively, the dimensionless star formation efficiency parameter and the local dynamical time. We employ $c_{*}=$ 0.01 in our simulations. If the mass of an SPH particle, $m_{\mathrm{SPH}}$, is more massive ${ }^{1}$ than $1.5 m_{*}$, where $m_{*}=0.5 m_{\mathrm{SPH}}^{\text {orig }}$, we compute the probability $\mathcal{P}_{\text {spawn }}$ for an SPH particle with which it spawns a new star particle of mass, $m_{*}$, during a time-step, $\Delta t$, as

$\mathcal{P}_{\text {spawn }}=\frac{m_{\mathrm{SPH}}}{m_{*}}\left[1-\exp \left(-\frac{c_{*} \Delta t}{t_{\mathrm{dyn}}}\right)\right]$.

When $m_{\mathrm{SPH}}$ is smaller than $1.5 m_{*}$, we compute the probability, $\mathcal{P}_{\text {conv }}$, with which the SPH particle is converted into a star particle of mass, $m_{\mathrm{SPH}}$, during $\Delta t$ as

$\mathcal{P}_{\text {conv }}=1-\exp \left(-\frac{c_{*} \Delta t}{t_{\text {dyn }}}\right)$,

in order to avoid the SPH particle mass from becoming too small.

A newly born star particle inherits smoothed (SPH-kernel averaged) chemical abundances of its parent gas particle as in Okamoto et al. (2005, 2010). Each star particle represents an SSP and its evolution is modelled as in Okamoto (2013). We employ the Chabrier IMF (Chabrier 2003) and use metallicity-dependent stellar lifetimes and chemical yields (Portinari, Chiosi \& Bressan 1998; Marigo 2001). We calculate Type II and Ia SN rates based on Portinari et al. (1998, see also Nagashima et al. 2005a). We track nine elements $(\mathrm{H}, \mathrm{He}, \mathrm{C}, \mathrm{N}, \mathrm{O}, \mathrm{Ne}, \mathrm{Mg}, \mathrm{Si}$, and $\mathrm{Fe}$ ) and we take $\mathrm{S}$ and $\mathrm{Ca}$ to be proportional to Si (Wiersma et al. 2009). To normalize chemical abundances of simulated galaxies by the solar values, we employ solar abundances compiled by Asplund et al. (2009), and thus $\mathrm{Z}_{\odot}$ $=0.0142$. On the other hand, we do not make any corrections to published $[\alpha / \mathrm{Fe}]$ or metallicity values, ${ }^{2}$ since we assume that observations measure relative abundances; applying the solar abundances by Asplund et al. (2009) for the observational estimates, however, does not affect our conclusions at all.

Stellar feedback is modelled as winds. Massive stars emit a large amount of ultraviolet photons in their early phases and then die as $\mathrm{SNe}$ II. We follow the luminosity evolution of each star particle

\footnotetext{
${ }^{1}$ In our simulations, the mass of an SPH particle can change by star formation and feedback.

${ }^{2}$ Observational estimates presented in this paper all assume the old canonical solar metallicity, $\mathrm{Z}_{\odot}=0.02$
}

and calculate how much photon energy and momentum are given to surrounding gas particles during a given time-step. The feedback due to radiation pressure is modelled as 'momentum-driven winds' (Oppenheimer \& Davé 2006), and we assume that the initial wind speed is scaled with the local dark matter velocity dispersion, $\sigma_{\mathrm{DM}}$. The wind mass loading is, hence, proportional to $\sigma_{\mathrm{DM}}^{-1}$ for a given momentum input. We also calculate, for each star particle, the number of stars that die as SNe II during a time-step. The energy released by $\mathrm{SNe}$ II is distributed to gas particles around the star particles. Feedback by SNe II is treated as 'energy-driven winds' (Okamoto et al. 2010). Again, the initial wind speed is scaled with $\sigma_{\mathrm{DM}}$ and therefore the wind mass loading is proportional to $\sigma_{\mathrm{DM}}^{-2}$. For gas particles which receive radiation pressure or $\mathrm{SN}$ energy, we attach probabilities with which they are added to winds. We then stochastically select wind particles and increase their velocities.

It is now widely accepted that stellar feedback alone cannot explain the bright end of the galaxy luminosity function and hence feedback processes that operate preferentially in massive galaxies are required (e.g. Benson et al. 2003). We introduce a simple, phenomenological analogue of the radio-mode AGN feedback (e.g. Bower et al. 2006; Croton et al. 2006; Okamoto et al. 2008). We simply assume that radiative cooling is suppressed for gas particles around which one-dimensional dark matter velocity dispersion is larger than $\sigma_{\text {th }}(z)$, where $z$ is redshift. We parametrize the functional form of $\sigma_{\text {th }}$ as $\sigma_{\text {th }}(z)=\sigma_{0}(1+z)^{\alpha}$. In order to reduce the cooling rate in massive haloes, as the radio-mode feedback does, we modify the cooling function, $\Lambda(T, Z)$, as

$\Lambda(T, Z, \sigma)= \begin{cases}\Lambda(T, Z) & \left(\sigma<\sigma_{\mathrm{th}}\right) \\ \Lambda(T, Z) \exp \left(-\frac{\sigma-\sigma_{\mathrm{th}}}{\beta \sigma_{\mathrm{th}}}\right) & \text { (otherwise) }\end{cases}$

where $T$ and $Z$ are, respectively, the gas temperature and the gas metallicity, and the parameter $\beta$ specifies how steeply the cooling is suppressed above $\sigma_{\text {th }}$. We adopt $\sigma_{0}=50 \mathrm{~km} \mathrm{~s}^{-1}, \alpha=0.75$, and $\beta=0.3$ in our reference simulation. This suppression of gas cooling is quite effective at low redshift. The cooling rate is halved in a halo with $\sigma \simeq 60 \mathrm{~km} \mathrm{~s}^{-1}$ at $z=0$ from the original cooling rate. We note that comparable results to this set of parameters can be obtained when we assume $\Lambda(T, Z, \sigma)=\Lambda(T, Z)$ for $\sigma<\sigma_{\text {th }}$ and $\Lambda(T, Z, \sigma)=0$ for $\sigma>\sigma_{\text {th }}$ with $\sigma_{0}=100 \mathrm{~km} \mathrm{~s}^{-1}$ and $\alpha=0.75$ (OSY14). The cooling is thus suppressed even in modest groups at $z=0$; the effect becomes almost negligible at high redshift ( $z \gtrsim 2$ ) owing to the redshift dependence in $\sigma_{\text {th }}(z)$. We refer to this suppression of the cooling in massive haloes as 'AGN feedback' in this paper. We also show the results for a simulation without AGN feedback, which is the simulation labelled ' $\mathrm{SN}+\mathrm{RP}$ ' in OSY14, for highlighting the effect of this process. The size of the simulation box, $\left(40 h^{-1} \mathrm{Mpc}\right)^{3}$, is smaller than that of the reference simulation.

To identify virialized dark matter haloes, we run the friends-offriends (FoF) group finder (Davis et al. 1985) with a linking length of 0.2 in units of the mean dark matter particle separation. Baryonic particles near dark matter particles that compose an FoF group are also regarded as members of the halo. We then use the SUBFIND algorithm (Springel et al. 2001) to identify gravitationally bound sub-groups of particles within each FoF halo. We regard a subgroup which consists of at least 32 particles and contains at least 10 star particles as a 'galaxy'. We then define radius of a galaxy in order to exclude diffusely distributing stellar components as done in Vogelsberger et al. (2013). We first calculate the stellar half-mass radius, $r_{\mathrm{h}}$, for each galaxy and define galaxy radius as $2 r_{\mathrm{h}}$. This definition of galaxy radius has almost no effect on the properties of low-mass galaxies, but it excludes some of the intracluster light for 
massive galaxies. Vogelsberger et al. (2013) show that this definition gives very similar results to more elaborate methods of excluding intracluster light.

We remove galaxies that do not contain more than 10 star particles within their $r$-band half-light radii from our sample. We compute chemical abundances of a galaxies as luminosity- or mass-weighted average of chemical abundances of the star particles within a specified radius (e.g. half-light radius and galaxy radius). We calculate the three-dimensional velocity dispersion of the star particles within the $r$-band half-light radius and then convert it into the one-dimensional velocity dispersion by multiplying a factor, $1 / \sqrt{3}$. We have confirmed that we obtain quantitatively similar results when we use projected half-light radius and line-of-sight velocity dispersion.

In addition, we analyse public data of the Illustris simulation (Illustris-1; Nelson et al. 2015) and the EAGLE simulation (RefL0100N1504; McAlpine et al. 2016) for comparison. We show the results whenever corresponding data are available. The details of the Illustris simulation are found in a series of papers (Vogelsberger et al. 2014a,b). Radius, chemical abundances, and velocity dispersion of Illustris galaxies are defined in the same way as in OSY14.

The EAGLE simulation is fully described in Schaye et al. (2015) and Crain et al. (2015). In the EAGLE simulation, galaxies are identified by using the FoF and SUBFIND algorithms as in OSY14 and Illustris. Stars gravitationally bound to a sub-group and within a spherical $30 \mathrm{kpc}$ aperture are used to define stellar mass, luminosity, and velocity dispersion of the galaxy (Schaye et al. 2015). The chemical abundances of individual elements of a galaxy in the public data base are, however, calculated as the mass-weighted average of chemical abundances of stars bound to a sub-halo. We will use these mass-weighted chemical abundances to compute abundance ratios of EAGLE galaxies.

There are some important differences between the reference, Illustris, and EAGLE simulations. While stellar feedback in the reference and Illustris simulations are implemented as winds, feedback energy is deposited locally as thermal energy in the EAGLE simulation. For AGN feedback, the reference simulation only has the radio-mode feedback. AGN feedback in the Illustris simulation has two modes: quasar and radio modes, while the EAGLE simulation only has the quasar-mode feedback.

\subsection{Identification of passive galaxies}

Morphological classification of simulated galaxies is difficult and could be affected by the limited numerical resolution. We hence focus on the chemical abundances of passive galaxies. We select galaxies whose specific SFRs are smaller than $10^{-11} \mathrm{yr}^{-1}$ as the passive galaxies. In Fig. 1, we show the colour-magnitude diagram of simulated galaxies in the reference simulation, the simulation without AGN feedback, the Illustris simulation, and the EAGLE simulation. For these plots, we use all the star particles belonging to a galaxy to compute luminosities and colours of the simulated galaxy and compare them with the colour-magnitude relation for SDSS red sequence measured by Skelton et al. (2009).

There is clearly a red sequence and a blue cloud in the reference, Illustris, and EAGLE simulations, while there are only a few red bright galaxies in the simulation without AGN feedback. In the reference simulation, there is a bridge between the red sequence and the blue cloud at $M_{r} \simeq-21$, where star-forming galaxies are being quenched by radio-mode feedback. We would be able to erase this feature by introducing quasar mode feedback that more quickly terminates star formation as in the Illustris and EAGLE simulations.

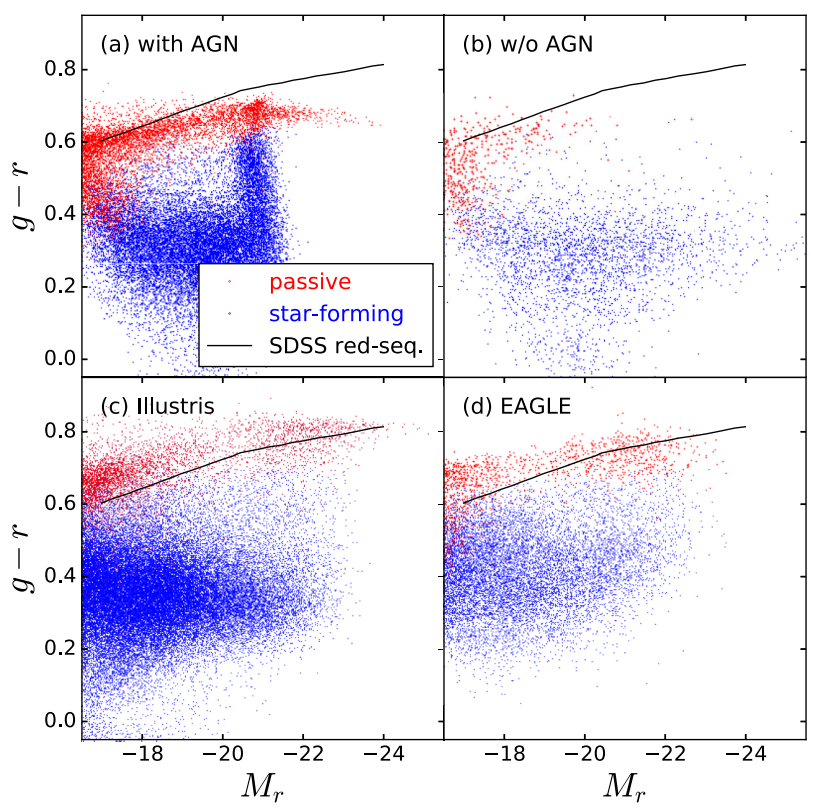

Figure 1. Colour-magnitude diagrams for the simulated galaxies. We plot $g-r$ colours for (a) the reference simulation, (b) the simulation without AGN feedback, (c) the Illustris simulation, and (d) the EAGLE simulation. We indicate passive galaxies and star-forming galaxies by red and blue dots, respectively. We also show the colour-magnitude relation for SDSS red sequence measured by Skelton, Bell \& Somerville (2009) by solid lines.

The colours of the passive galaxies in the reference simulation are somewhat bluer than the SDSS red sequence. We find that metallicities of passive galaxies would need to be doubled in order to agree with observations. The chemical yields assumed here, however, can explain the chemical abundances of the Local Group satellites (Okamoto et al. 2010) and Milky Way-mass galaxies (Nagashima \& Okamoto 2006). Passive galaxies in the Illustris simulation are slightly redder than the SDSS red sequence, reflecting higher metallicities than those in the reference simulation as we will show later. The EAGLE simulation reproduces the red sequence reasonably well, although the faint-end slope is slightly shallower than the data (see also Trayford et al. 2015 for EAGLE galaxies). Galaxies with specific SFRs smaller than $10^{-11} \mathrm{yr}^{-1}$ lie on the red sequence in all simulations and this simple criterion successfully selects passive galaxies.

\section{RESULTS}

\subsection{The $\alpha$-to-Fe ratios of simulated galaxies}

In Fig. 2, we plot the $[\alpha / \mathrm{Fe}]-\sigma$ relations for galaxies in the reference simulation, the simulation without AGN feedback, and the EAGLE simulation. We do not show Illustris galaxies because the public data of the Illustris simulation do not include abundances of $\alpha$ elements. We also show the observational estimates by Thomas et al. (2010) for the MOSES sample (Schawinski et al. 2009; Thomas et al. 2010). We only use passive galaxies in MOSES to compare with the passive galaxies in the simulations. For the simulations by OSY14, we show luminosity weighted $[\alpha / \mathrm{Fe}]$, while for the EAGLE simulation, we show mass-weighted $[\alpha / \mathrm{Fe}]$.

From convergence studies in OSY14, we find that ages and metallicities of the simulated galaxies in the reference simulation and that without AGN feedback are reliable down to $M_{*} \sim 10^{9.5} \mathrm{M}_{\odot}$; this stellar mass corresponds to the velocity dispersion, 


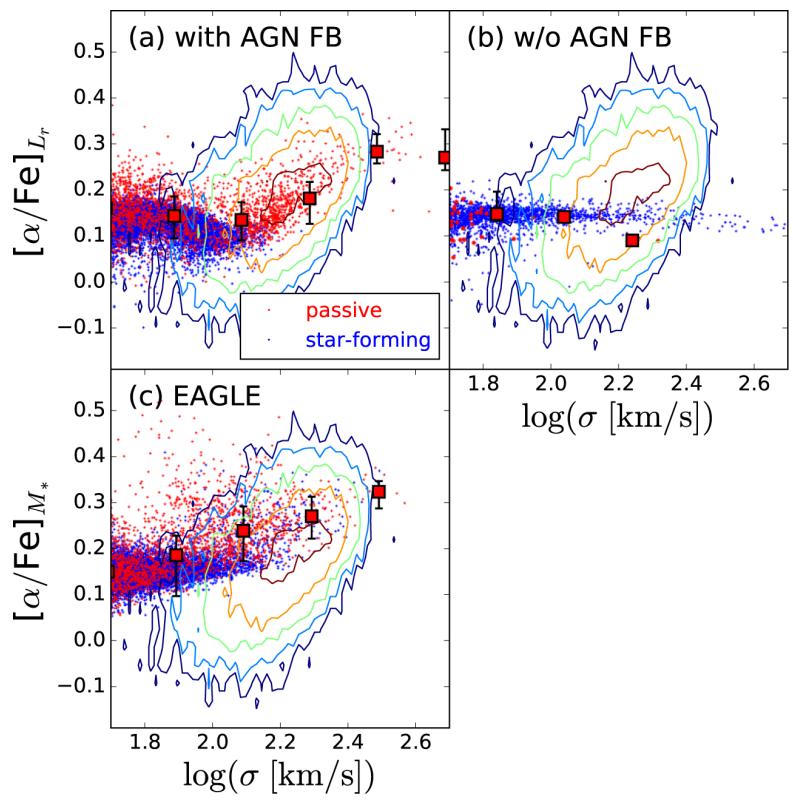

Figure 2. Luminosity-weighted $[\alpha / \mathrm{Fe}]$ versus velocity dispersion of galaxies in (a) the reference simulation and (b) the simulation without AGN feedback. We also show the mass-weighted $[\alpha / \mathrm{Fe}]$ of Eagle galaxies in panel (c). Passive and star-forming galaxies are, respectively, indicated by the red and blue dots. The median $[\alpha / \mathrm{Fe}]$ in a velocity bin and 16th to 84th percentile distribution for the passive galaxies are indicated by a red square with an error bar. Observational estimates for SDSS passive earlytype galaxies (Thomas et al. 2010) are shown by the contours.

$\sigma \sim 10^{1.6} \mathrm{~km} \mathrm{~s}^{-1}$. We hence expect that the $[\alpha / \mathrm{Fe}]$ relations shown in Fig. 2 are not affected by the numerical resolution. We have also confirmed that a result obtained by a simulation with higher resolution (but with a smaller box size) agrees with that in Fig. 2.

The reference simulation reproduces the observational trend for high- $\sigma$ galaxies well; $[\alpha / \mathrm{Fe}]$ of the passive galaxies increases with the velocity dispersion for $\sigma \gtrsim 100 \mathrm{~km} \mathrm{~s}^{-1}$. Below $\sigma \sim 100 \mathrm{~km} \mathrm{~s}^{-1}$, $[\alpha / \mathrm{Fe}]$ becomes nearly constant, while the observational trend continues at least down to $\sigma \sim 10^{1.7} \mathrm{~km} \mathrm{~s}^{-1}$ (e.g. Spolaor et al. 2010; Johansson et al. 2012). The star-forming galaxies, in the reference simulation, exist below $\sigma \sim 10^{2.1} \mathrm{~km} \mathrm{~s}^{-1}$ and have slightly lower $[\alpha / \mathrm{Fe}]$ than the passive galaxies. In the simulation without AGN feedback, $[\alpha / \mathrm{Fe}]$ does not depend on the velocity dispersion, and the value is consistent with that for $\alpha \lesssim 100 \mathrm{~km} \mathrm{~s}^{-1}$ in the reference simulation. Segers et al. (2016b) showed similar results with the EAGLE simulation, that is, $[\alpha / \mathrm{Fe}]$ increases with stellar mass for galaxies with $\mathrm{M}_{*}>10^{10.5} \mathrm{M}_{\odot}$ when $\mathrm{AGN}$ feedback is included, while it is roughly constant without AGN feedback. As expected from the results by Segers et al. (2016b), the EAGLE simulation also shows an increasing $[\alpha / \mathrm{Fe}]$ for passive galaxies with velocity dispersion, while the relation extends to lower velocity dispersion and its slope is somewhat shallower than in the reference simulation. It is interesting that almost all simulated galaxies (both passive and star forming) have $[\alpha / \mathrm{Fe}]>0$. This is partly because low-mass simulated galaxies are too old compared with their observed counterparts (OSY14). Top-light IMFs for low-mass galaxies and/or a delayed distribution of SNe Ia with an increased normalization compared with the DTD currently assumed would also decrease $[\alpha / \mathrm{Fe}]$ of low-mass galaxies without changing $[\alpha / \mathrm{Fe}]$ of massive galaxies.

To understand the origin of the $[\alpha / \mathrm{Fe}]-\sigma$ relation for $\sigma \gtrsim$ $100 \mathrm{~km} \mathrm{~s}^{-1}$ in the reference simulation, we investigate the star formation histories and the evolution of the stellar $[\alpha / \mathrm{Fe}]$ for galaxies having different velocity dispersion at $z=0$. We show the

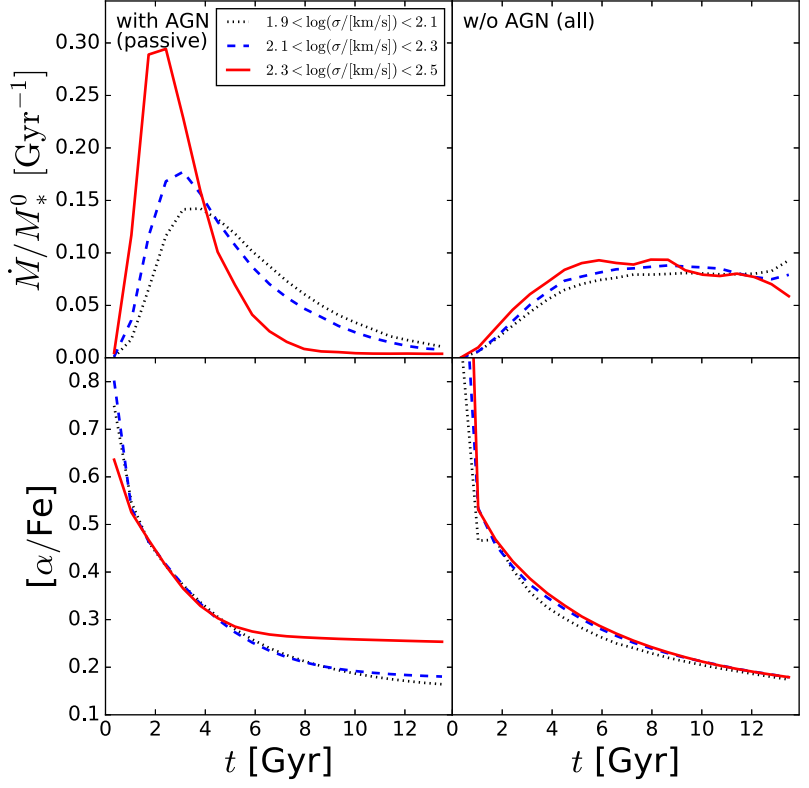

Figure 3. Upper panels: mean specific SFRs (normalized by the stellar mass at $z=0$ ) as a function of cosmic time in three velocity bins at $z=$ $0: 10^{1.9}<\sigma /\left(\mathrm{km} \mathrm{s}^{-1}\right)<10^{2.1}$ (black dotted), $10^{2.1}<\sigma /\left(\mathrm{km} \mathrm{s}^{-1}\right)<10^{2.3}$ (blue dashed), and $10^{2.3}<\sigma /\left(\mathrm{km} \mathrm{s}^{-1}\right)<10^{2.5}$ (red solid). The formation histories of the stars within the half-light radius at $z=0$ are shown. Leftand right-hand panels, respectively, show the reference simulation and the simulation without AGN feedback. We only use passive galaxies for the reference simulation, while all the galaxies in a velocity bin are used for the simulation without AGN feedback, which are mostly star forming. Lower panels: same as the upper panel but for $[\alpha / \mathrm{Fe}]$. We here show the massweighted $[\alpha / \mathrm{Fe}]$ of the stars that have formed by time $t$.

normalized formation histories and the time evolution of $[\alpha / \mathrm{Fe}]$ of stars within the half-light radii at $z=0$, dividing galaxies into three velocity bins in Fig. 3. In the reference simulation, galaxies with high $\sigma$ have a peak in the star formation history at an earlier epoch and a shorter star formation time-scale than those with lower $\sigma$. This is a direct consequence of our AGN feedback model that suppresses gas cooling earlier in haloes with higher velocity dispersion. As shown in Fig. 3, the longer star formation time-scale for lower $\sigma$ galaxies reduces $[\alpha / \mathrm{Fe}]$ with time as discussed in Segers et al. (2016b). On the other hand, in the simulation without AGN feedback, the normalized star formation histories are almost identical in all the velocity bins. As a result, the evolution of $[\alpha / \mathrm{Fe}]$ becomes independent of the velocity dispersions at $z=0$.

\subsection{The stellar metallicities of simulated galaxies}

The reference simulation reproduces well the gas phase metallicity of the star-forming galaxies (OSY14). We now examine the stellar metallicities of the simulated galaxies. In the left-hand panels of Fig. 4, we show luminosity-weighted stellar mass-stellar metallicity relations (MZRs) of galaxies in the reference simulation, in the simulation without AGN feedback, in the Illustris simulation, and in the EAGLE simulation.

For the simulations by OSY14 and the Illustris simulation, we calculate the $r$-band luminosity-weighted average of metallicities of stars bound to a sub-halo and within a galaxy radius defined as twice the stellar half-mass radius. For the EAGLE simulation, we use all stars bound to a sub-halo to estimate the metallicity instead of applying a $30 \mathrm{kpc}$ aperture because, at $M_{*} \gtrsim 10^{11} \mathrm{M}_{\odot}$, the galaxy radius in the reference and Illustris simulations is larger than $30 \mathrm{kpc}$ 


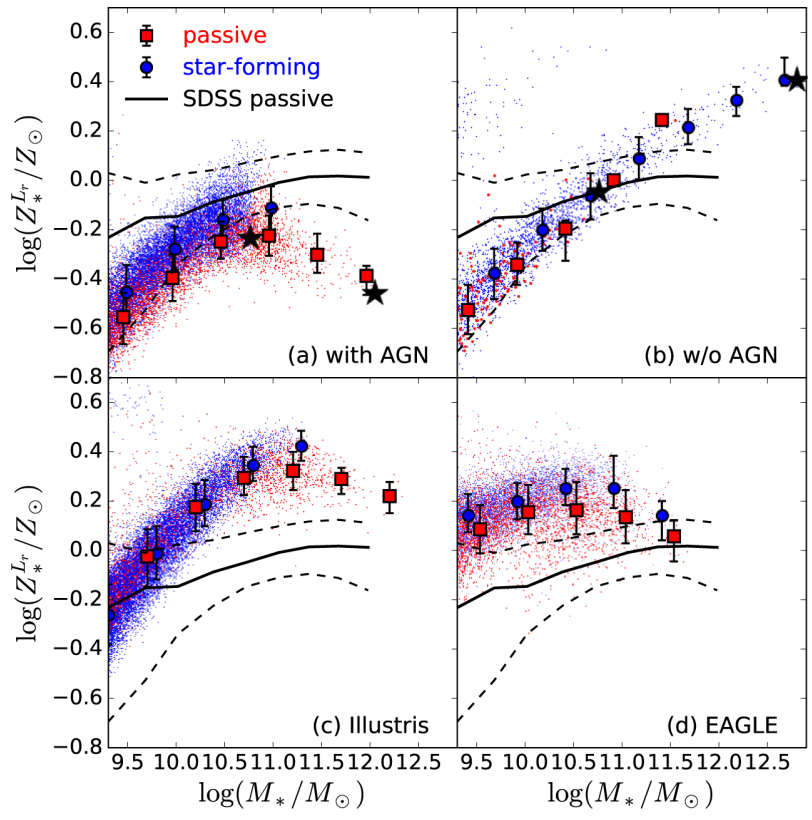

Figure 4. Stellar mass-stellar metallicity relation for the simulated galaxies. We show $r$-band luminosity-weighted metallicities for (a) the reference simulation, (b) the simulation without AGN feedback, (c) the Illustris simulation, and (d) the EAGLE simulation. Passive and star-forming galaxies are, respectively, indicated by the red and blue dots. The squares show the median metallicities in the mass bins for the passive galaxies and the circles for the star-forming galaxies. The observational estimates for SDSS passive galaxies are shown by the black lines; the solid line indicates the median of the distribution and the dotted lines the 16th and 84th percentiles, where we apply the aperture correction as described in Appendix A. The black stars in panel (a) indicate two passive central galaxies for which we investigate the enrichment histories later in Fig. 5. In panel (b), we also highlight two (star-forming) galaxies by black stars, for which we show the enrichment histories in Fig. 5 too.

and monotonically increases with stellar mass. We also show the MZR of SDSS passive galaxies, where we correct the observed metallicity for each galaxy from an aperture value to a global one (see Appendix A).

In the reference simulation, the metallicities of passive galaxies are lower than the observational estimate. For low-mass galaxies with $M_{*} \lesssim 10^{10.5} \mathrm{M}_{\odot}$, the MZR is steeper than the observational estimate, whereas more massive galaxies, $M_{*} \gtrsim 10^{10.5} \mathrm{M}_{\odot}$, show a trend of decreasing metallicity with increasing stellar mass. A similar trend is found for Illustris galaxies, although they on average have higher metallicities than the galaxies in the reference simulation. The higher metallicities of the Illustris galaxies compared with those in the reference simulation are explained by the fact that, in Illustris, the metallicity of a newly created wind particle is chosen to be 40 per cent of the metallicity of the ambient interstellar medium, while, in OSY14, all the metal mass attached to a gas particle is ejected when the particle is launched as a wind. The mass range where metallicity negatively correlates with stellar mass is the mass range where AGN feedback operates. In fact, the stellar metallicities in the simulation without AGN feedback monotonically increase with the stellar mass.

We find that galaxies in the EAGLE simulation have much flatter MZR than those in the reference and Illustris simulations. The slope is more consistent with the observational estimate for the passive galaxies. The massive EAGLE galaxies, however, show a weak trend of decreasing metallicity with increasing stellar mass as those in the reference and the Illustris simulations. This trend is consistent with the finding of Segers et al. (2016a) using the EAGLE simulation. They showed that the fraction of stellar mass contributed by recycled stellar-ejecta increases with stellar mass for less massive galaxies and this fraction turns over at $\mathrm{M}_{*} \sim 10^{10.5} \mathrm{M}_{\odot}$ owing to AGN feedback. This feature seems to be common in simulations that invoke AGN feedback. We, however, note that the metallicities of passive galaxies monotonically increase with stellar mass when we employ a $30 \mathrm{kpc}$ aperture for the EAGLE simulation. The same applies to the Illustris and reference simulations. We must be careful about metallicity gradients within galaxies when comparing simulated and observed metallicities.

A similar problem has been reported using semi-analytic models. Pipino et al. (2009) successfully produce an increasing $[\alpha / \mathrm{Fe}]$ with galaxy stellar mass by invoking radio-mode AGN feedback. They, however, find that the AGN feedback erases a positive correlation between stellar metallicity and velocity dispersion. McCarthy et al. (2010) show that hydrodynamic simulations including quasar-mode AGN feedback predict central group galaxies that have too low metallicity compared with observational data, while, in simulations without AGN feedback, the metallicities of central galaxies become higher than observed. We will investigate the origin of this problem later.

For less massive galaxies with $M_{*} \lesssim 10^{11} \mathrm{M}_{\odot}$, the luminosityweighted metallicities of passive galaxies in the reference simulation are, on average, slightly lower than those of star-forming galaxies. The EAGLE simulation shows a similar trend. In the Illustris simulation, passive galaxies have comparable metallicities to star-forming galaxies. Although measuring stellar metallicities of star-forming galaxies is difficult, tentative observational estimates suggest that the luminosity-weighted metallicities of passive galaxies are significantly higher than those of star-forming galaxies (Peng, Maiolino \& Cochrane 2015). If passive galaxies do in fact have much higher metallicities than star-forming galaxies, we would need to consider a process which preferentially enriches passive galaxies.

To understand the reason why simulations with AGN feedback generally yield inversely correlated MZR, we investigate formation and enrichment histories of galaxies. From the reference simulation, we select a central passive galaxy at the knee of the MZR and the most massive galaxy. The masses and metallicities of these galaxies at $z=0$ are $\left(M_{*, 0}, Z_{*, 0}^{L_{r}}\right) \simeq\left(5.7 \times 10^{10} \mathrm{M}_{\odot}, 0.59 \mathrm{Z}_{\odot}\right)$ and $\left(1.1 \times 10^{12} \mathrm{M}_{\odot}, 0.35 \mathrm{Z}_{\odot}\right)$. For comparison, we pick two central starforming galaxies, $\left(M_{*, 0}, Z_{*, 0}^{L_{r}}\right) \simeq\left(5.7 \times 10^{10} \mathrm{M}_{\odot}, 0.9 \mathrm{Z}_{\odot}\right)$ and $(6.3$ $\left.\times 10^{12} \mathrm{M}_{\odot}, 2.6 \mathrm{Z}_{\odot}\right)$, from the simulation without AGN feedback; the latter is the most massive galaxy in the simulation. While the most massive galaxy in the simulation without AGN feedback is more massive than that in the reference simulation, its halo mass, $M_{\text {vir }} \simeq 2 \times 10^{14} \mathrm{M}_{\odot}$, is smaller than that in the reference simulation, $M_{\mathrm{vir}} \simeq 4 \times 10^{14} \mathrm{M}_{\odot}$.

In Fig. 5, we show formation and enrichment histories of in situ and accreted stars within these galaxies. In situ stars are the stars born in the main progenitor of each galaxy and accreted stars are those born in sub-clumps.

The main progenitor of the less massive galaxy in the reference simulation continues its star formation down to low redshift. On the other hand, AGN feedback quenches the formation of in situ stars in the most massive galaxy at early times $(t \sim 3 \mathrm{Gyr})$. Consequently, this galaxy is dominated by accreted stars at $z=0$. The mass fractions of in situ stars in the less massive and the most massive galaxies are 0.97 and 0.066 at $z=0$, respectively. The metallicities of the less massive and most massive galaxies thus, respectively, reflect the metallicities of in situ and accreted stars. 


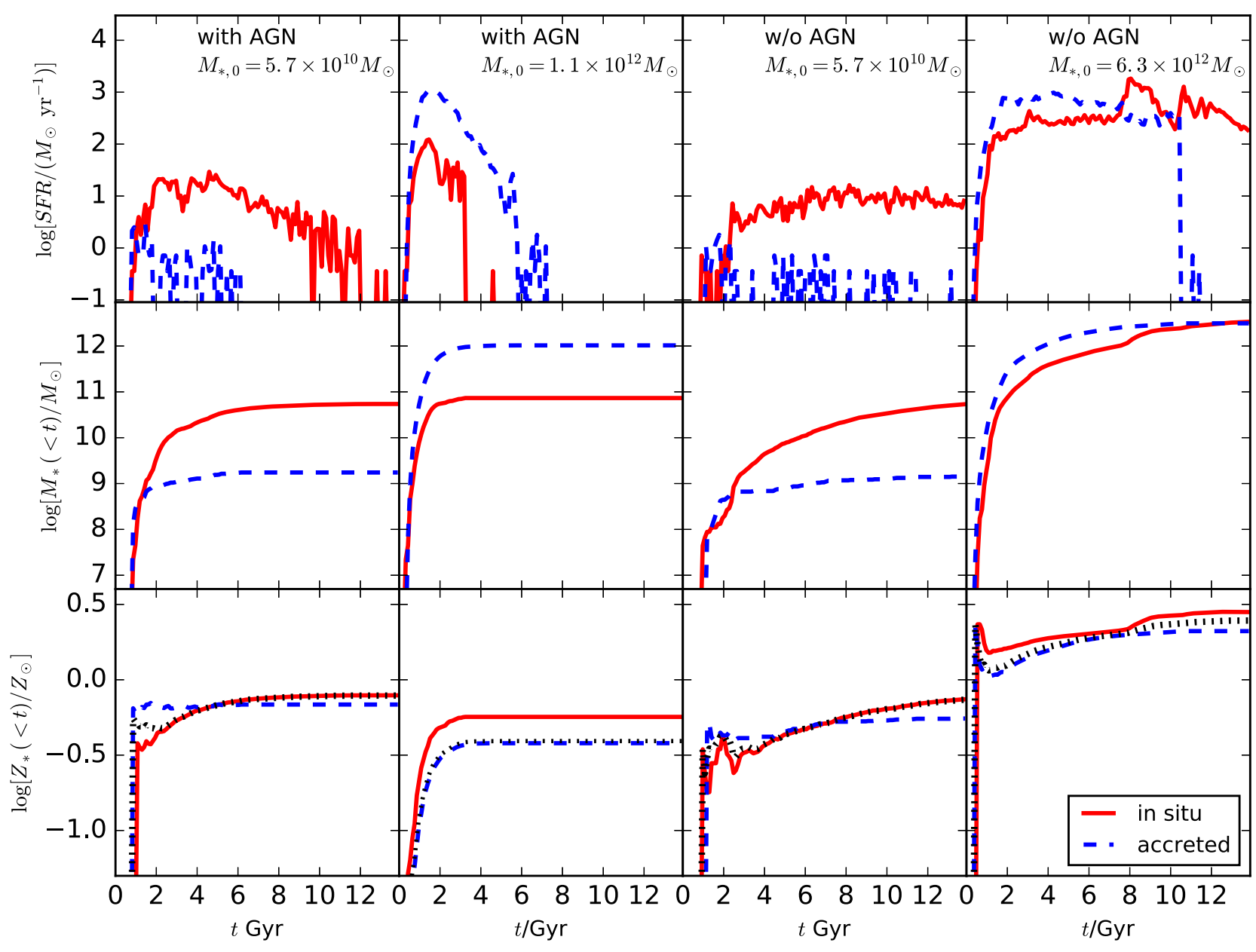

Figure 5. Formation and enrichment histories of selected central galaxies. In the first and second columns, we show two central passive galaxies in the reference simulation with stellar masses of $5.7 \times 10^{7}$ and $1.1 \times 10^{12} \mathrm{M}_{\odot}$, respectively. In the third and fourth columns, we show galaxies in the simulation without AGN feedback. The less massive galaxy has similar stellar mass to that in the reference simulation $\left(M_{*} \simeq 5.7 \times 10^{7} \mathrm{M}_{\odot}\right)$. The galaxy shown in the fourth column is the most massive galaxy $\left(M_{*} \simeq 6.3 \times 10^{12} \mathrm{M}_{\odot}\right)$ in this simulation. These galaxies are indicated by the stars in the panels (a) and (b) of Fig. 4 . From top to bottom, we show SFRs, masses of stars that have formed by time $t$, and mass-weighted average metallicities of stars that have formed by time $t$. Formation and enrichment histories of stars which formed in the main progenitor of each galaxy (in situ) are indicated by the red solid lines, while those of stars born in sub-clumps are shown by the blue dashed lines. In the bottom panels, we show total (in situ + accreted) metallicities by the black dotted lines.

The main progenitor of the most massive galaxy always has a higher velocity dispersion than that of the less massive galaxy and hence it has fewer outflows per star formed than the main progenitor of the less massive galaxy. ${ }^{3}$ We, however, find that in situ stars in the most massive galaxy have lower metallicity than in the less massive galaxy. The earlier suppression of gas cooling by AGN feedback in larger haloes prevents massive galaxies from recapturing enriched outflows and renders their metallicities lower than in their less massive counterparts as discussed by De Lucia et al. (2006).

In the simulation without AGN feedback, the formation of in situ stars of both galaxies continues to $z=0$. Even without AGN feedback, the fraction of in situ stars decreases with increasing galaxy mass as indicated by Naab et al. (2014). The metallicities of both in situ and accreted stars of the most massive galaxy in this simulation are much higher than those in the reference simulation.

\footnotetext{
${ }^{3}$ Wind mass loading scales with $\sigma^{-2}$ for SN-driven winds and $\sigma^{-1}$ for radiation-driven winds in our simulations.
}

The low metallicity of accreted stars of the most massive galaxy in the reference simulation suggests that sub-clumps in high-density regions also undergo AGN feedback. In fact, the SFR of the accreted stars in the most massive galaxy of the reference simulation shows a clear sign of early (but later than in situ stars) quenching.

\section{SUMMARY AND DISCUSSION}

We have analysed the chemical abundances of simulated passive galaxies. The reference simulation has previously been shown to reproduce many observed properties of galaxies at $z \leq 4$, such as the stellar mass function, SFRs, ages, and gas phase metallicities of star-forming galaxies (OSY14). We have also analysed publicly available data of two independent cosmological simulations: the Illustris (Vogelsberger et al. 2014b) and the EAGLE (Schaye et al. 2015) simulations. We define passive galaxies as those whose specific SFRs are smaller than $10^{-11} \mathrm{yr}^{-1}$. This definition selects galaxies that lie on a red sequence.

We find that the $[\alpha / \mathrm{Fe}]-\sigma$ relation of passive galaxies in the reference simulation is consistent with observations, that is, $[\alpha / \mathrm{Fe}]$ increases with velocity dispersion for $\sigma>100 \mathrm{~km} \mathrm{~s}^{-1}$. Passive 
galaxies in the EAGLE simulation also show the same trend. In the reference simulation, star formation in galaxies with high velocity dispersion today is quenched earlier by AGN feedback than in galaxies with lower velocity dispersion. Consequently, galaxies with higher velocity dispersions have shorter star formation time-scales and older stellar ages than those with lower velocity dispersion. This process explains the trend in the $[\alpha / \mathrm{Fe}]-\sigma$ relation at $\sigma \gtrsim$ $100 \mathrm{~km} \mathrm{~s}^{-1}$. For less massive galaxies with $\sigma \lesssim 100 \mathrm{~km} \mathrm{~s}^{-1}$, which are not affected by AGN feedback, $[\alpha / \mathrm{Fe}]$ is nearly constant; this behaviour might be inconsistent with the observations which suggest that the $[\alpha / \mathrm{Fe}]-\sigma$ trend exists at least for $\sigma \gtrsim 10^{1.7} \mathrm{~km} \mathrm{~s}^{-1}$ (Spolaor et al. 2010; Johansson et al. 2012).

Earlier quenching of star formation in more massive galaxies by AGN feedback, however, also decreases the stellar metallicity for increasing stellar mass. In spite of different implementations of physical processes, three independent simulations which invoke AGN feedback produce inversely correlated stellar-mass-to-metallicity relations for massive galaxies, which contradict observational estimates.

We investigate the enrichment and formation histories of passive galaxies with different masses in the reference simulation. We find that metallicities of both in situ and accreted stars in massive galaxies are lower than those in less massive galaxies because of early quenching of gas cooling in massive galaxies, which prevents enriched winds from falling back later. The shorter formation timescale of the accreted stars in the massive galaxy compared with its counterpart in the simulation without AGN feedback suggests that not only the main progenitor but also other progenitors are subject to AGN feedback. Simultaneously explaining the increasing $\alpha$-to-Fe ratio with velocity dispersion (or stellar mass) and increasing stellar metallicity with stellar mass is therefore fundamentally difficult within the current framework of galaxy formation. One possible solution would be the introduction of a variable IMF which becomes more top-heavy for a higher SFR as suggested by Calura \& Menci (2009) and Gargiulo et al. (2015) and previously suggested on independent grounds by Baugh et al. (2005) and Lacey et al. (2016).

Luminosity-weighted metallicities of passive galaxies in the reference simulation are, on average, lower than those of star-forming galaxies. Passive galaxies in the EAGLE simulation also have slightly lower metallicities than star-forming galaxies for a given stellar mass. In the Illustris simulation, passive galaxies have similar luminosity-weighted metallicities to star-forming galaxies. Tentative observational estimates suggest that the luminosity-weighted metallicities of passive galaxies are significantly higher than those of star-forming galaxies for a given stellar mass (Peng et al. 2015). None of the three simulations can produce this metallicity gap. A process which preferentially enriches passive galaxies would explain this observational result. More accurate measurements of stellar metallicity will thus place strong constraints on the galaxy formation theory.

\section{ACKNOWLEDGEMENTS}

We thank Masato Onodera for his helpful comments on the observational data. Numerical simulations were carried out with Cray XC30 in CfCA at NAOJ. TO acknowledges the financial support of Japan Society for the Promotion of Science (JSPS) Grant-in-Aid for Young Scientists (B: 224740112) and The Ministry of Education, Culture, Sports, Science and Technology (MEXT) KAKENHI Grant (16H01085). MN acknowledges the financial support of JSPS Grant-in-Aid (B: 25287041). CSF acknowledges ERC Advanced
Investigator grant COSMIWAY. This work was supported in part by STFC Consolidated grant ST/L00075X/1. We also thank the Illustris collaboration for making data publicly available. We acknowledge the Virgo Consortium for making their simulation data available. The EAGLE simulations were performed using the DiRAC-2 facility at Durham, managed by the ICC, and the PRACE facility Curie based in France at TGCC, CEA, Bruyères-le-Chátel.

\section{REFERENCES}

Arimoto N., Yoshii Y., 1987, A\&A, 173, 23

Arrigoni M., Trager S. C., Somerville R. S., Gibson B. K., 2010, MNRAS, 402, 173

Asplund M., Grevesse N., Sauval A. J., Scott P., 2009, ARA\&A, 47, 481

Baugh C. M., Lacey C. G., Frenk C. S., Granato G. L., Silva L., Bressan A., Benson A. J., Cole S., 2005, MNRAS, 356, 1191

Bender R., Burstein D., Faber S. M., 1993, ApJ, 411, 153

Benson A. J., Bower R. G., Frenk C. S., Lacey C. G., Baugh C. M., Cole S., 2003, ApJ, 599, 38

Bower R. G., Benson A. J., Malbon R., Helly J. C., Frenk C. S., Baugh C. M., Cole S., Lacey C. G., 2006, MNRAS, 370, 645

Brinchmann J., Charlot S., White S. D. M., Tremonti C., Kauffmann G., Heckman T., Brinkmann J., 2004, MNRAS, 351, 1151

Calura F., Menci N., 2009, MNRAS, 400, 1347

Calura F., Menci N., 2011, MNRAS, 413, L1

Chabrier G., 2003, PASP, 115, 763

Conroy C., Graves G. J., van Dokkum P. G., 2013, ApJ, 780, 33

Crain R. A. et al., 2015, MNRAS, 450, 1937

Croton D. J. et al., 2006, MNRAS, 365, 11

Davé R., Oppenheimer B. D., Finlator K., 2011, MNRAS, 415, 11

Davis M., Efstathiou G., Frenk C. S., White S. D. M., 1985, ApJ, 292, 371

De Lucia G., Borgani S., 2012, MNRAS, 426, L61

De Lucia G., Springel V., White S. D. M., Croton D., Kauffmann G., 2006, MNRAS, 366, 499

Di Matteo T., Springel V., Hernquist L., 2005, Nature, 433, 604

Faber S. M., 1973, ApJ, 179, 731

Gallazzi A., Charlot S., Brinchmann J., White S. D. M., Tremonti C. A., 2005, MNRAS, 362, 41

Gallazzi A., Charlot S., Brinchmann J., White S. D. M., 2006, MNRAS, 370,1106

Gargiulo I. D. et al., 2015, MNRAS, 446, 3820

Johansson J., Thomas D., Maraston C., 2012, MNRAS, 421, 1908

Jørgensen I., 1999, MNRAS, 306, 607

Kauffmann G., Charlot S., 1998, MNRAS, 294, 705

Lacey C. G. et al., 2016, MNRAS, 462, 3854

Larson R. B., 1975, MNRAS, 173, 671

McAlpine S. et al., 2016, Astron. Comput., 15, 72

McCarthy I. G. et al., 2010, MNRAS, 406, 822

Marigo P., 2001, A\&A, 370, 194

Matteucci F., 1994, A\&A, 288, 57

Naab T. et al., 2014, MNRAS, 444, 3357

Nagashima M., Okamoto T., 2006, ApJ, 643, 863

Nagashima M., Lacey C. G., Baugh C. M., Frenk C. S., Cole S., 2005a, MNRAS, 358, 1247

Nagashima M., Lacey C. G., Okamoto T., Baugh C. M., Frenk C. S., Cole S., 2005b, MNRAS, 363, L31

Nelson D. et al., 2015, Astron. Comput., 13, 12

O’Connell R. W., 1976, ApJ, 206, 370

Okamoto T., 2013, MNRAS, 428, 718

Okamoto T., Eke V. R., Frenk C. S., Jenkins A., 2005, MNRAS, 363, 1299

Okamoto T., Nemmen R. S., Bower R. G., 2008, MNRAS, 385, 161

Okamoto T., Frenk C. S., Jenkins A., Theuns T., 2010, MNRAS, 406, 208

Okamoto T., Shimizu I., Yoshida N., 2014, PASJ, 66, 70 (OSY14)

Oppenheimer B. D., Davé R., 2006, MNRAS, 373, 1265

Peng Y., Maiolino R., Cochrane R., 2015, Nature, 521, 192

Pipino A., Devriendt J. E. G., Thomas D., Silk J., Kaviraj S., 2009, A\&A, 505, 1075 
Planck Collaboration XVI, 2014, A\&A, 571, A16

Portinari L., Chiosi C., Bressan A., 1998, A\&A, 334, 505

Rawle T. D., Smith R. J., Lucey J. R., 2010, MNRAS, 401, 852

Romeo Velonà A. D., Sommer-Larsen J., Napolitano N. R., Antonuccio-

Delogu V., Cielo S., Gavignaud I., Meza A., 2013, ApJ, 770, 155

Salpeter E. E., 1955, ApJ, 121, 161

Schawinski K. et al., 2009, MNRAS, 396, 818

Segers M. C., Crain R. A., Schaye J., Bower R. G., Furlong M., Schaller M., Theuns T., 2016a, MNRAS, 456, 1235

Segers M. C., Schaye J., Bower R. G., Crain R. A., Schaller M., Theuns T., 2016b, MNRAS, 461, L102

Schaye J. et al., 2015, MNRAS, 446, 521

Shen S., Mo H. J., White S. D. M., Blanton M. R., Kauffmann G., Voges W., Brinkmann J., Csabai I., 2003, MNRAS, 343, 978

Sijacki D., Springel V., Di Matteo T., Hernquist L., 2007, MNRAS, 380, 877

Skelton R. E., Bell E. F., Somerville R. S., 2009, ApJ, 699, L9

Spolaor M., Kobayashi C., Forbes D. A., Couch W. J., Hau G. K. T., 2010, MNRAS, 408, 272

Springel V., White S. D. M., Tormen G., Kauffmann G., 2001, MNRAS, 328,726

Taylor P., Kobayashi C., 2015, MNRAS, 448, 1835

Thomas D., 1999, MNRAS, 306, 655

Thomas D., Greggio L., Bender R., 1999, MNRAS, 302, 537

Thomas D., Maraston C., Schawinski K., Sarzi M., Silk J., 2010, MNRAS, 404, 1775

Trayford J. W. et al., 2015, MNRAS, 452, 2879

Vogelsberger M., Genel S., Sijacki D., Torrey P., Springel V., Hernquist L., 2013, MNRAS, 436, 3031

Vogelsberger M. et al., 2014a, MNRAS, 444, 1518

Vogelsberger M. et al., 2014b, Nature, 509, 177

Wiersma R. P. C., Schaye J., Theuns T., Dalla Vecchia C., Tornatore L., 2009, MNRAS, 399, 574

Worthey G., Faber S. M., Gonzalez J. J., 1992, ApJ, 398, 69

Wu H., Shao Z., Mo H. J., Xia X., Deng Z., 2005, ApJ, 622, 244

Yates R. M., Henriques B., Thomas P. A., Kauffmann G., Johansson J., White S. D. M., 2013, MNRAS, 435, 3500

\section{APPENDIX A: TOTAL METALLICITIES OF SDSS PASSIVE GALAXIES}

To obtain an observational estimate for stellar metallicities of passive galaxies, we take metallicity and stellar mass estimates of the SDSS DR4 galaxies from Gallazzi et al. (2005). We only use galaxies with $\mathrm{S} / \mathrm{N}>20$. We select galaxies whose specific SFRs are smaller than $10^{-11} \mathrm{yr}^{-1}$ as candidates of passive galaxies by taking SFR estimates from Brinchmann et al. (2004). We then remove galaxies that are classified as star forming or composite from the passive galaxy candidates. The median MZR of the passive galaxies obtained this way is indicated by the dashed line in Fig. A1. This relation is consistent with the MZR of early-type galaxies in Gallazzi et al. (2006) and that of passive galaxies in Peng et al. (2015).

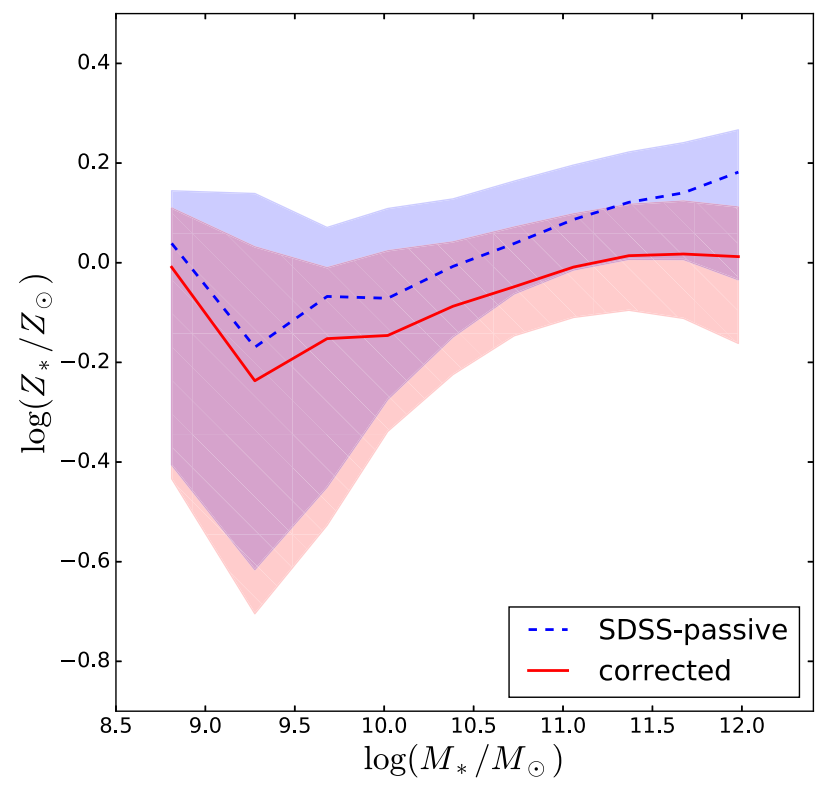

Figure A1. Mass-metallicity relation for SDSS passive galaxies. Stellar metallicities and masses are taken from Gallazzi et al. (2005). The median MZR obtained from the aperture values is shown by the blue dashed line. We correct the observed metallicity for each galaxy from an aperture value to a global one by using redshift of each galaxy and assuming a fixed metallicity gradient. The median MZR obtained this way is indicated by the red solid line. The shaded regions represent the 16th to 84th distributions. Note that we assume $\mathrm{Z}_{\odot}=0.02$ as in Gallazzi et al. (2005).

Early-type galaxies are known to have radial metallicity gradients (e.g. Wu et al. 2005; Rawle, Smith \& Lucey 2010). Since the original spectra were measured in 1.5 arcsec radius fibre aperture in SDSS, we correct the observed metallicities from aperture values to mean global values assuming a uniform metallicity gradient, $\mathrm{d} \log Z / \mathrm{d} \log r=-0.15$, based on Rawle et al. (2010) and by using the redshift of each galaxy. We here assume that the light profile in each galaxy follows an $r^{1 / 4}$ raw and the median size-mass relation for early-type galaxies by Shen et al. (2003). The corrected median MZR is shown by the solid line in Fig. A1.

This paper has been typeset from a $\mathrm{T}_{\mathrm{E}} \mathrm{X} / \mathrm{L} \mathrm{T} \mathrm{E} \mathrm{X}$ file prepared by the author. 